ARTICLE

Received 13 May 2014 | Accepted 15 Dec 2014 | Published 20 Jan 2015

DOI: $10.1038 /$ ncomms7102

OPEN

\title{
Origins of multicellular evolvability in snowflake yeast
}

William C. Ratcliff', Johnathon D. Fankhauser ${ }^{2}$, David W. Rogers ${ }^{3}$, Duncan Greig ${ }^{3,4}$ \& Michael Travisano 5,6

Complex life has arisen through a series of 'major transitions' in which collectives of formerly autonomous individuals evolve into a single, integrated organism. A key step in this process is the origin of higher-level evolvability, but little is known about how higher-level entities originate and gain the capacity to evolve as an individual. Here we report a single mutation that not only creates a new level of biological organization, but also potentiates higher-level evolvability. Disrupting the transcription factor ACE2 in Saccharomyces cerevisiae prevents mother-daughter cell separation, generating multicellular 'snowflake' yeast. Snowflake yeast develop through deterministic rules that produce geometrically defined clusters that preclude genetic conflict and display a high broad-sense heritability for multicellular traits; as a result they are preadapted to multicellular adaptation. This work demonstrates that simple microevolutionary changes can have profound macroevolutionary consequences, and suggests that the formation of clonally developing clusters may often be the first step to multicellularity.

\footnotetext{
${ }^{1}$ School of Biology, Georgia Institute of Technology, Atlanta, Georgia 30332-0230, USA. ${ }^{2}$ Plant Biology, University of Minnesota, St Paul, Minnesota 55108, USA. ${ }^{3}$ Max Planck Institute for Evolutionary Biology, 24306 Plön, Germany. ${ }^{4}$ Department of Genetics, Evolution, and Environment, University College London, London WC1N 6BT, UK. ${ }^{5}$ Department of Ecology, Evolution and Behavior, University of Minnesota, St Paul, Minnesota 55108, USA. ${ }^{6}$ The BioTechnology Institute, University of Minnesota, St Paul, Minnesota 55108, USA. Correspondence and requests for materials should be addressed to W.C.R. (email: william.ratcliff@biology.gatech.edu).
} 
O ne of the most conspicuous features of Earth's organisms is their complexity. In their paradigm-defining synthesis, John Maynard Smith and Eörs Szathmáry identified key steps (termed 'major transitions') through which complexity evolves ${ }^{1}$. The multilevel selection hypothesis for major transitions posits a two-step process: first, a solitary ancestor evolves to form collectives $^{2}$, then selection shifts to the collective level ${ }^{1,3,4}$. Biological complexity arises as a result of adaptation in the new, higher-level organismal unit ${ }^{1,5-7}$. The multilevel selection hypothesis has strong historical and theoretical support for many of the major transitions in evolution (for example, origins of cells, chromosomes, eukaryotes, multicellularity and eusocial superorganismality ${ }^{1,8-10}$ ), but understanding how higher-level entities originate and gain the ability evolve as Darwinian Individuals remains a challenge ${ }^{11,12}$.

The conditions required for higher-level adaptation are stringent ${ }^{13}$. First, collectives must be capable of reproducing ${ }^{6,7,12}$. Second, collectives must have the properties required for Darwinian evolution, namely they must vary in their collectivelevel traits, this variation must be heritable, and these collectivelevel traits must affect fitness 9 ,12-14. Finally, internal conflicts must be minimized. Collective-level adaptations can easily be undermined by within-collective (lower-level) evolution ${ }^{15-19}$, particularly if higher-level adaptations reduce the fitness of lower-level parts (for example, cellular division of labour). Collectives that undergo a bottleneck during reproduction limit lower-level genetic diversity, reducing the potential for conflict between the fitness of the collective and its constituents ${ }^{1,16,20-26}$. How do incipient multicellular organisms meet these criteria and gain the capacity to evolve as Darwinian individuals?

We can glean several clues from life's successful and failed transitions in individuality. Collectives of like individuals ('fraternal' transitions) ${ }^{27}$ are thought to be important for the evolution of chromosomes from independent replicators, multicellular organisms from solitary cells and eusocial 'super organisms' from asocial multicellular ancestors. These collectives faced the classic problems of group selection, namely that clusterlevel adaptation requires that the strength of among-collective selection exceed the strength of within-collective selection ${ }^{28}$. Heritable diversity among lower-level units within collectives is a key factor determining the relative strength of lower- versus higher-level selection ${ }^{29}$. Clonal collectives align the fitness interests of lower-level units, and as a result the primary way for a lower-level unit (for example, a cell) to increase its fitness is by enhancing the collective's fitness (for example, a multicellular organism). Most multicellular- and super-organisms have solved this problem by producing propagules that develop through a unicellular genetic bottleneck, limiting migration of lower-level units between collectives $1,16,21-23,25,30,31$. The single-cell bottleneck and subsequent clonal development is thus a key trait facilitating the evolution of higher-level complexity in fraternal transitions. Two widely studied social organisms, the slime mold Dictylostelium discoideium and bacterium Myxococcus xanthus, appear stuck in the transition to multicellularity, despite ample time to evolve multicellular complexity ( $>400 \mathrm{Myr}$ ago for the Dictyostelid cellular slime molds $s^{32}$ and $>650 \mathrm{Myr}$ ago for the myxobacteria ${ }^{33}$ ). While both organisms possess multicellular life histories that include cellular division of labour, neither life cycle includes a single-cell bottleneck, and genetic conflict is rampant ${ }^{18,34,35}$. This conflict can select for adaptations that limit the diversity of cells within collectives (for example, policing ${ }^{36,37}$, greenbeards ${ }^{38}$ ), but these mechanisms are not as effective or evolutionarily durable as the single-cell bottleneck.

Collectives of unlike individuals ('egalitarian' transitions) ${ }^{27}$ are thought to be critical for the evolution of cells from populations of replicating molecules, chromosomes from unlinked replicators and eukaryotes from a symbiotic pair. Multispecies collectives are common in nature, ranging in size and complexity from communities to pairs of species interacting synergistically (for example, cross-feeding microbial consortia). Multispecies collectives can possess a substantial fitness advantage relative to solitary competitors ${ }^{39-41}$, and thus provide a rich substrate for selection among collectives to facilitate higher-level adaptation. Yet few multispecies collectives have made the transition to a higher level of individuality. Multispecies collectives encounter all of the challenges faced by uni-species collectives (see above), as well as the added difficulty of regenerating a collective from two or more distinct genetic backgrounds. In the absence of a developmental mechanism that ensure partner fidelity across multiple generations of the collective (for example, codispersal ${ }^{42,43}$, vertical transmission ${ }^{44}$ or partner choice $\left.^{45}\right)$, the heritability of collective-level traits collective is limited. The importance of transmission mode is illustrated by differences in symbiosis: only vertically transmitted symbionts have become a part of a new, higher-level organism (for example, the multiple origins of plastids ${ }^{46}$ ). In contrast, horizontally transmitted symbionts (for example, legumes and Rhizobium, bobtail squid and Vibrio) have failed to make the transition from symbiont to organelle. Taken together, it is clear that collectives that successfully transit to a higher level of individuality possess heritable multicellular traits that selection may act on, and exhibit little within-group conflict.

Here we use the transition from uni- to multicellularity as a model to investigate the origin of higher-level evolvability. We previously evolved multicellularity in the yeast Saccharomyces cerevisiae. Starting with a single diploid clone of strain Y55 (a unicellular yeast), we selected for rapid settling through liquid media in 10 replicate populations. Within 60 daily transfers, multicellular 'snowflake' yeast evolved in all 10 populations, displacing their unicellular ancestors. Snowflake yeast result from daughter cells remaining attached to their parent cells after mitosis. Snowflake yeast display a key emergent property: as clusters grow larger, tension among cells increases until it exceeds the tensile strength of a cell-cell connection, resulting in the release of a multicellular propagule ${ }^{5}$. Once clusters have evolved, they readily become a unit of selection, as whole clusters either settle rapidly enough to survive, or fail to do so and perish. As a result of this shift to cluster-level selection, we observe extensive cluster-level adaptation, including the evolution of larger size, elevated apoptosis and more spherical, hydrodynamic clusters ${ }^{5,47}$. While the evolution of larger clusters reduces their number in the population, our cluster-level effective population size remains large, minimizing the role of genetic drift. Even in one of our largest cluster-forming strains from 60 days, the effective population size $\left(N_{\mathrm{e}}\right)$ of clusters is still $9.4 \times 10^{5}$ (calculated as the harmonic mean of population size variation over each day).

We follow first principles in examining the origin of multicellular evolvability in our yeast model system. We first characterize the genetic basis of multicellularity in snowflake yeast and then describe their three-dimensional developmental pattern mathematically. We next determine how this growth form affects the within-collective genetic variation, and examine its implications for the evolution of within-cluster genetic conflict and multicellular heritability. We find that the snowflake yeast developmental pattern imbues clusters with a high degree of multicellular evolvability, demonstrating that seemingly simple molecular changes can have profound macroevolutionary consequences.

\section{Results}

Genetic basis of the snowflake yeast developmental pattern. Comparing the gene expression between the unicellular Y55 
ancestor and an early (7-day) snowflake yeast, we found that 1,035 genes were significantly differentially expressed $8 \mathrm{~h}$ after transfer. Of these, only 143 genes differed by more than twofold. Of the 10 most downregulated genes (Table 1), seven (CTS1, DSE4, DSE2, SUN4, DSE1, SCW11 and AMN1) are regulated by the trans-acting transcription factor ACE2 (marked with triangles in Fig. 1a; Tables 1-3), suggesting that the native function of ACE2 is disrupted in early snowflake yeast. These seven most downregulated genes are involved in daughter cell separation, many acting directly to degrade the bud neck septum $^{48-50}$, and prior work has shown that ACE2 knockouts form cellular clusters ${ }^{48,51,52}$. We next sequenced ACE2 from 10 independently evolved lineages of snowflake yeast (populations 1-10 from Ratcliff et al. ${ }^{5}$ ). Non-synonymous mutations were detected in five populations, either causing protein truncation (populations 3 and 6) or amino-acid substitution in (or adjacent to) ACE2's zinc finger-binding domain (populations 1, 2, 8; Fig. $1 \mathrm{~b}$ and Table 2). In all cases, both ACE2 alleles in the diploid yeast were identical, suggesting that the mutant $A C E 2$ allele was made homozygous by gene conversion, a common occurrence in yeast ${ }^{53}$. These mutations likely lead to non (or less)-functional ACE2p. We confirmed that a loss of ACE2 functionality would develop the snowflake phenotype by constructing a diploid ace2::NATMX4/ace2::NATMX4 knockout in the unicellular ancestor (Fig. 1c). Further, we found that complementation of an experimentally evolved snowflake yeast (population 1 , codon $645^{\mathrm{val}}$ " asp) with a single copy of functional ACE2 linked to KANMX4 resulted in reversion to unicellularity (Fig. 1d). The snowflake yeast phenotype can therefore be produced by the disruption of a single gene.

Modelling the snowflake yeast developmental pattern. Snowflake yeast clusters grow by daughter cell adhesion to maternal cells (Fig. 2a). This results in a mathematically tractable growth form where, if cells reproduce at the same rate, the number of cells distance $x$ from the basal cell follows Pascal's triangle (Fig. 2b). The number of cells distance $x$ from the basal cell $c_{d}(x)$ can be calculated after $d$ doublings with the binomial expression $\left(\begin{array}{l}d \\ x\end{array}\right)$. In practice, all cells do not divide synchronously, so we modified the above binomial to include a partial doubling: the reproduction of fraction $s$ cells. We therefore can describe the structure of a snowflake yeast cluster of any size by predicting the number of cells distance $x$ from the basal cell (see Supplementary Methods for a complete derivation of the model):

$$
c_{d}(x)+s\left(\left(\begin{array}{c}
d+1 \\
x
\end{array}\right)-\left(\begin{array}{l}
d \\
x
\end{array}\right)\right)
$$

To assess our model's description of snowflake yeast growth form, we compared the predicted number of cells of each distance from the basal cell $(0,1,2,3 \ldots x)$ with experimentally obtained counts. To generate this prediction, we calculated the number of complete doublings from the basal cell, $d$, as $\log _{2}$ (cell number), rounded down. We also calculated $s$, the fraction of cells within the cluster that reproduced from the last complete doubling, as described above. Inputting the values of $d$ and $s$ into our model, we obtained the expected number of cells at each distance $x$ from the basal cell. We then compared the actual number of cells at each distance from the basal cell, determined empirically (Fig. 2c), to the number predicted by our model. Our model accurately described the growth form of snowflake yeast clusters $\left(\mathrm{F}_{9,311}=574.7, P<0.0001\right.$, main effect of predicted number of cells per generation in an analysis of covariance with observed number of cells per generation as the response variable and yeast strain as the cofactor, overall $r^{2}=0.94$. The main effect

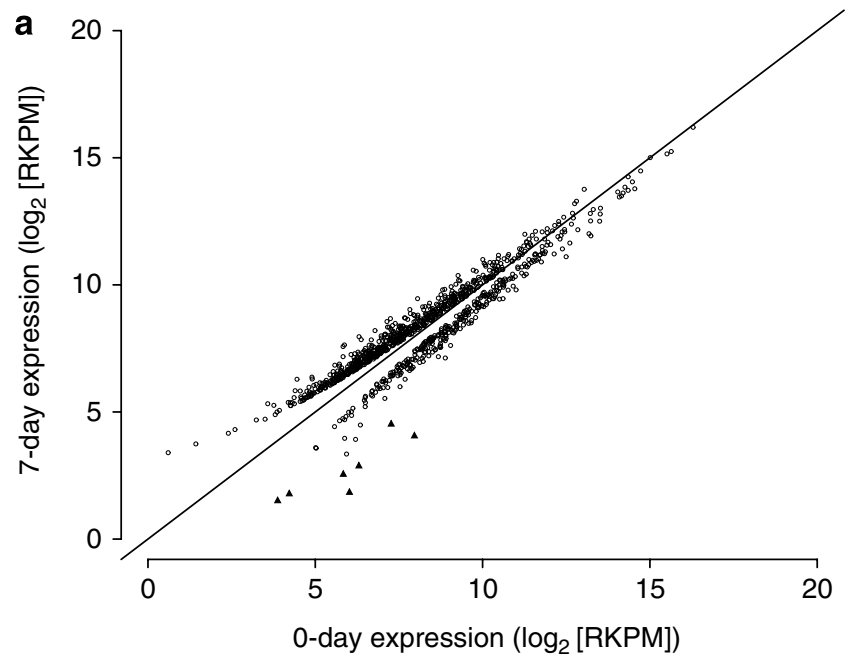

b
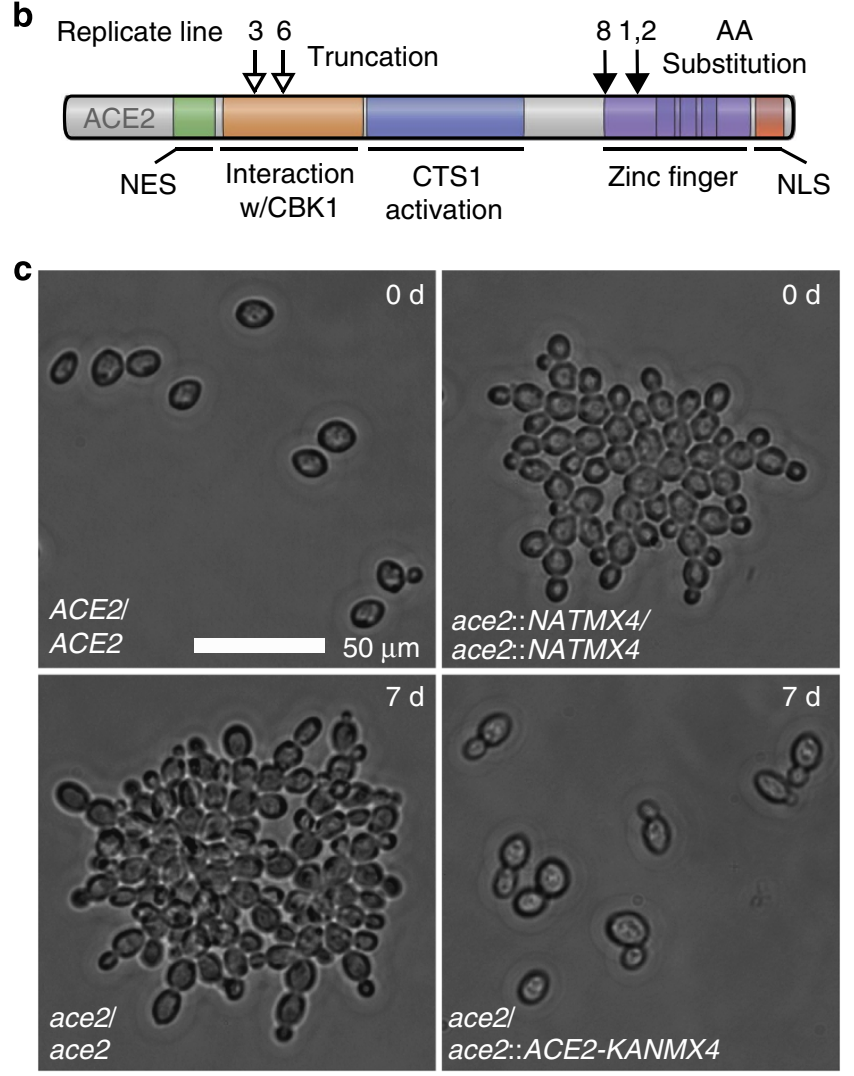

Figure 1 | Genetic basis of multicellularity. (a) 1,035 genes were differentially expressed between the unicellular ancestor and an early (7-day) snowflake yeast. Of the 10 most downregulated genes, seven are regulated by the transcription factor ACE2 (triangles). (b) Non-synonymous mutations were found in the ACE2 of 5/10 lineages (open arrows designate nonsense mutations, closed arrows designate missense mutations).

(c) Knocking out ACE2 in the unicellular ancestor (upper right) results in snowflake yeast, while functionally complementing the 7-day strain (lower right) restores unicellularity.

of yeast strain and the interaction with the cofactor were not significant Fig. 2d).

The growth form of snowflake yeast conforms to what we would expect if newly produced cells remain attached to their parent cell, and it takes each cell a similar amount of time to reproduce. Our analysis implies that the snowflake yeast growth 


\begin{tabular}{|c|c|c|c|}
\hline Gene & $\begin{array}{c}\text { Fold } \\
\text { change }\end{array}$ & $\begin{array}{l}\text { FDR-adjusted } \\
\quad P \text { value }\end{array}$ & Function \\
\hline SCW11 & -16.5 & $6.22 \cdot 10^{-12}$ & $\begin{array}{l}\text { Cell wall protein with similarity to glucanases; may play a role in conjugation during mating } \\
\text { based on its regulation by Ste12p. }\end{array}$ \\
\hline CTS1 & -13.6 & $2.86 \cdot 10^{-43}$ & $\begin{array}{l}\text { Endochitinase, required for cell separation after mitosis; transcriptional activation during the } \\
\text { G1 phase of the cell cycle is mediated by transcription factor Ace2p. }\end{array}$ \\
\hline DSE1 & -9.7 & $1.21 \cdot 10^{-12}$ & $\begin{array}{l}\text { Daughter cell-specific protein, may regulate crosstalk between the mating and filamentation pathways; } \\
\text { deletion affects cell separation after division and sensitivity to alpha-factor and drugs affecting the cell wa }\end{array}$ \\
\hline AMN1 & -8.8 & $5.55 \cdot 10^{-9}$ & $\begin{array}{l}\text { Protein required for daughter cell separation, multiple mitotic checkpoints, and chromosome stability; } \\
\text { contains } 12 \text { degenerate leucin10-rich repeat motifs; expression is induced by the Mitotic Exit Network. }\end{array}$ \\
\hline DSE2 & -6 & $7.10 \cdot 10^{-20}$ & $\begin{array}{l}\text { Daughter cell-specific secreted protein with similarity to glucanases, degrades cell wall from the } \\
\text { daughter side causing daughter to separate from mother; expression is repressed by cAMP. }\end{array}$ \\
\hline $\mathrm{IZH} 4$ & -5.3 & $1.34 \cdot 10^{-7}$ & $\begin{array}{l}\text { Membrane protein involved in zinc ion homeostasis, member of the four-protein IZH family, expression } \\
\text { induced by fatty acids and altered zinc levels; deletion reduces sensitivity to excess zinc; possible } \\
\text { role in sterol metabolism. }\end{array}$ \\
\hline YNL277W-A & -4.9 & 0.013919 & Putative protein of unknown function. \\
\hline YFR057W & -4.7 & 0.040354 & Putative protein of unknown function. \\
\hline DSE4 & -4.3 & $6.32 \cdot 10^{-08}$ & $\begin{array}{l}\text { Daughter cell-specific secreted protein with similarity to glucanases, degrades cell wall from the } \\
\text { daughter side causing daughter to separate from mother. }\end{array}$ \\
\hline SUN4 & -3.3 & $3.92 \cdot 10^{-05}$ & Cell wall protein related to glucanases, possibly involved in cell wall septation; member of the SUN family. \\
\hline
\end{tabular}

cAMP, cyclic AMP; FDR, false discovery rate

Genes involved in daughter cell separation have been bolded. Gene function obtained from the Saccharomyces Genome Database.

\begin{tabular}{|c|c|c|}
\hline Population & Codon position & Amino-acid change \\
\hline 1 & 645 & Val »Asp \\
\hline 2 & 645 & Val » Asp \\
\hline 3 & 194 & Gln » Stop \\
\hline 6 & 238 & Gln » Stop \\
\hline 8 & 610 & Cys » Ser \\
\hline
\end{tabular}

form does not change during the course of 227 days of experimental evolution ( $\sim 1,135$ generations), despite substantial changes in cell size, cluster size and settling velocity ${ }^{47}$. Using ideal snowflake yeast clusters generated from the model above, we examine key evolutionary consequences of this developmental pattern.

Propagules always experience a unicellular genetic bottleneck. Each time a propagule separates from a parent cluster, it goes through a single-cell genetic bottleneck, even if the propagule is multicellular. Consider the separation of any two connected cells in a snowflake yeast cluster. This will cause fragmentation and production of a new cluster (Fig. 3a). Due to the way that snowflake yeast grow, one of the two resulting clusters will always be one of the 'branches' of cells from the parent cluster (except in the case where it is a single cell), and this branch will always contain a single cell at its base (denoted by a dashed outline and 0 ' annotation in Fig. 3a). All other cells in the propagule are clonal descendants of this basal cell. Thus all propagules experience single-cell genetic bottlenecks, despite multicellular propagation. This mode of reproduction greatly limits within-cluster genetic diversity and acts as a conflict mediator ${ }^{54}$, facilitating multicellular adaptation ${ }^{1,16,20-26}$.

Snowflake yeast rapidly purge within-cluster genetic variation. Selection in our experimental populations readily acts on the properties of whole clusters, such as settling speed. Consider the evolution of larger cells, a trait that evolves in our experimental populations (Fig. 4a) and is predicted to increase cluster size and settling speed by $\sim 45 \%$ (ref. 47). Cluster-level selection favours the groups composed entirely of large-celled yeast, but clusterlevel selection alone cannot easily increase the frequency of a large-cell mutation when the mutant is rare. This is because the benefit of faster settling is shared with a greater number of wildtype (small) cells in the same cluster. How then does selection favour initially rare mutations that confer only a cluster-level fitness benefit?

Cluster-level selection can easily favour cellular traits that provide no within-group fitness advantage (such as large cell size) when genetic variation is effectively partitioned between groups. To examine how the dynamics of cluster developmental mode affect the genetic composition of propagules, we model ideal snowflake yeast clusters and contrast this with cellular aggregates. Cellular aggregates, which can be thought of as biofilm-like structures (similar to yeast flocs), do not necessarily undergo a genetic bottleneck when producing propagules. We assume propagules are up to half of the parent cluster's size, and that the mutation leading to larger cells only occurs once within a cluster, after which it is passed on to daughter cells within the cluster. Consider a 16-celled cluster containing 50\% small and large cells. Snowflake yeast, whose propagules pass through a unicellular genetic bottleneck, completely segregate genetic variation among offspring, while only $0.6 \%$ of the propagules produced by aggregative clusters contain just the large-celled mutant (Fig. 5a). We arrive at this result as follows: in this snowflake yeast cluster, all cell-cell connections that are broken must be between either two mutant cells (yielding a mutant-only propagule), a mutant cell and a wild-type cell (yielding two equalsized uniclonal mutant and wild-type clusters) or between two wild-type cells (yielding a wild-type-only propagule). Within aggregative clusters, $n$ cells (up to half the size of the cluster) are chosen randomly for inclusion in propagules. The number of ways the aggregative cluster can produce $100 \%$ large-celled propagules is $n_{l} ! / x !\left(n_{l}-x\right) !$, where $n_{1}$ is the number of large cells in the cluster, and $x$ is propagule size. Similarly, the probability of getting $100 \%$ small cells is $n_{s} ! / x !\left(n_{s}-x\right)$ !, where $n_{\mathrm{s}}$ is the number of small cells in the cluster. Finally, the total 
Table 3 | Primers used in this study.

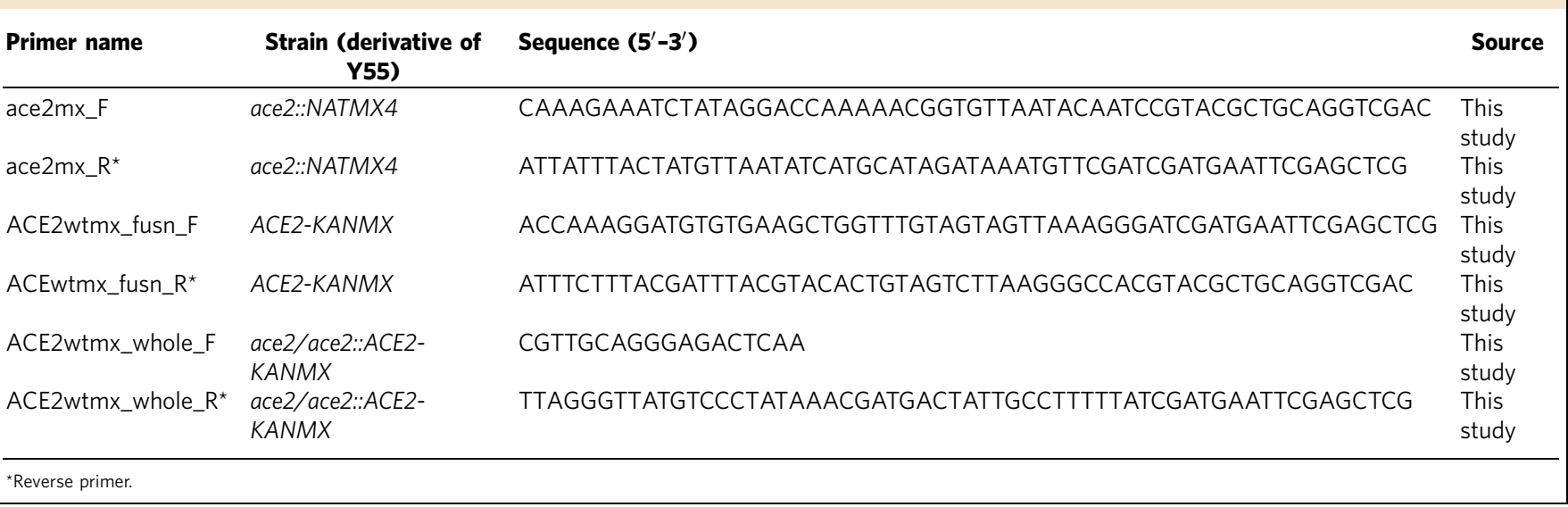

a

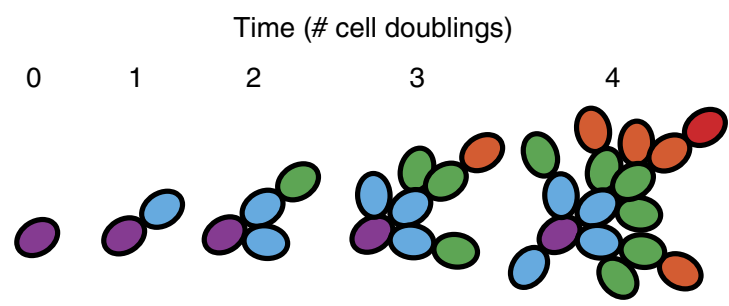

b

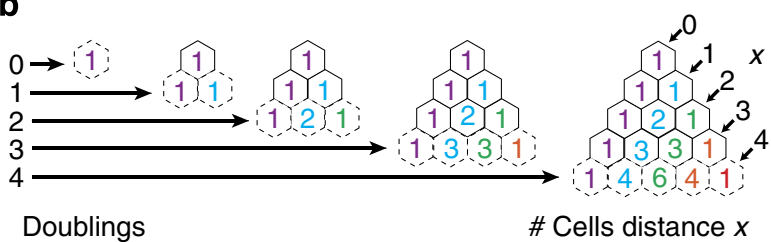

C

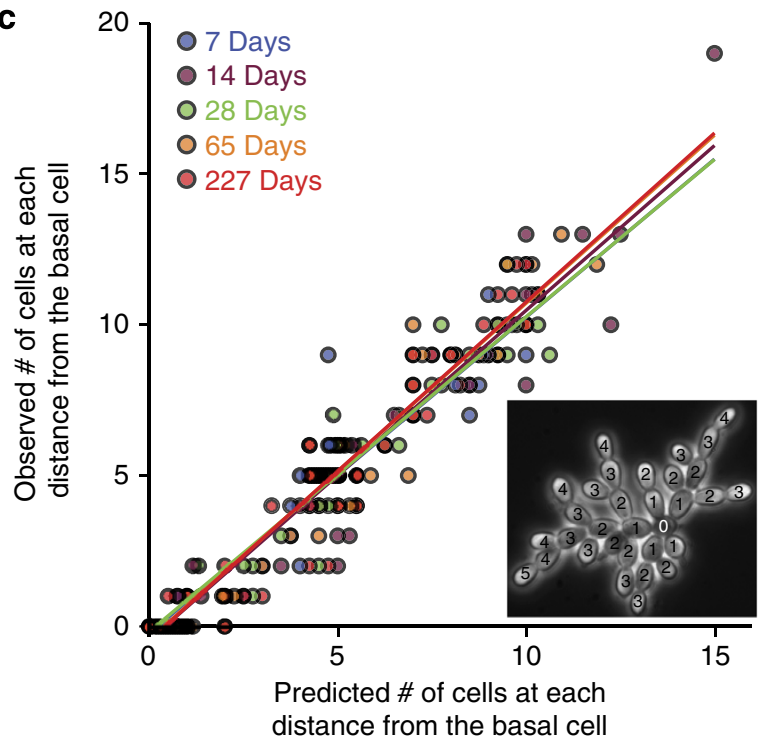

Figure 2 | Modelling the snowflake yeast body plan. (a) Cluster growth from a single cell, with each distance from the basal cell (purple) labelled a different colour. (b) Pascal's triangle describes the number of cells distance $x$ from the basal cell. (c) The model accurately predicts the growth form of snowflake yeast. Despite a $45 \%$ increase in settling speed over 227 days of evolution ${ }^{21}$, we find no differences in the growth form of any of our snowflake yeast isolates. Inset: a cluster with each cell's position from the basal labeled. number of non-clonal propagules can be determined by $\left(n_{l} ! /(x-1) !\left(n_{l}-x+1\right) !\right)\left(n_{\mathrm{s}} ! /(x-1) !\left(n_{\mathrm{s}}-x+1\right) !\right)(x-1)$. We generate the result in Fig. 5 a by dividing the total number of clonal propagules from the total number of non-clonal propagules for offspring size 1-8.

The model presented in Fig. 5a considered mutants at high frequency. Rare mutants provide an even stronger advantage for snowflake yeast. A mutant-only propagule will be produced when fragmentation occurs between either the mutant and a wild-type cell, or two mutant cells. When all breakages between cells are equally likely, this is $n_{\mathrm{m}} /\left(n_{\mathrm{tot}}-1\right)$, where $n_{\mathrm{m}}$ and $n_{\text {tot }}$ are the number of mutant and total cells in the cluster, respectively. With the snowflake yeast body plan, the probability of producing a uniclonal mutant propagule is therefore approximately the same as the mutant frequency in the cluster (Fig. 5b). For aggregate clusters, the probability that a randomly produced propagule from an aggregate will be mutant only is:

$$
\frac{2^{n_{\mathrm{m}}}-1}{\sum_{i=1}^{n_{\text {tot }} / 2} \frac{n_{\text {tot }} !}{\left(n_{\text {tot }}-i\right) !(i !)}}
$$

This is simply the fraction of total unique propagules of size 1 to $n_{\text {tot }} / 2$ (denominator) that will contain only mutant cells (numerator). The probability of producing a mutant-only propagule is negligible $\left(\sim 10^{-39}\right.$ for a 256 -cell cluster containing $50 \%$ mutant cells, Fig. 5b), and declines exponentially with reduced mutant frequency. As a result, segregation of the mutant into its own clusters will be extremely rare, limiting the capacity for selection to act on the multicellular phenotype of individual mutations (a limitation similar to that imposed by 'blending' modes of inheritance ${ }^{55}$ ).

Rare mutant cells have little chance of founding a uniclonal propagule, even in snowflake yeast (Fig. 5b). However, when wild-type-only propagules are produced, the frequency of mutant cells in the snowflake parent cluster is increased. As a result, the probability that the next propagule to be produced will be mutant-only $\left(p_{\mathrm{m}}\right)$ increases with each wild-type-only propagule that is produced (Fig. 5c),

$$
p_{\mathrm{m}}=\frac{n_{\mathrm{m}} /\left(n_{\mathrm{tot}}-1\right)}{(1-\beta)^{q}}
$$

where $\beta$ is the size of each propagule relative to the whole cluster before division and $q$ is the number of wild-type-only propagules produced. The snowflake body plan thus ensures that all mutants will eventually form uniclonal propagules, regardless of their initial frequency in a cluster (Fig. 5c), allowing the cluster-level 


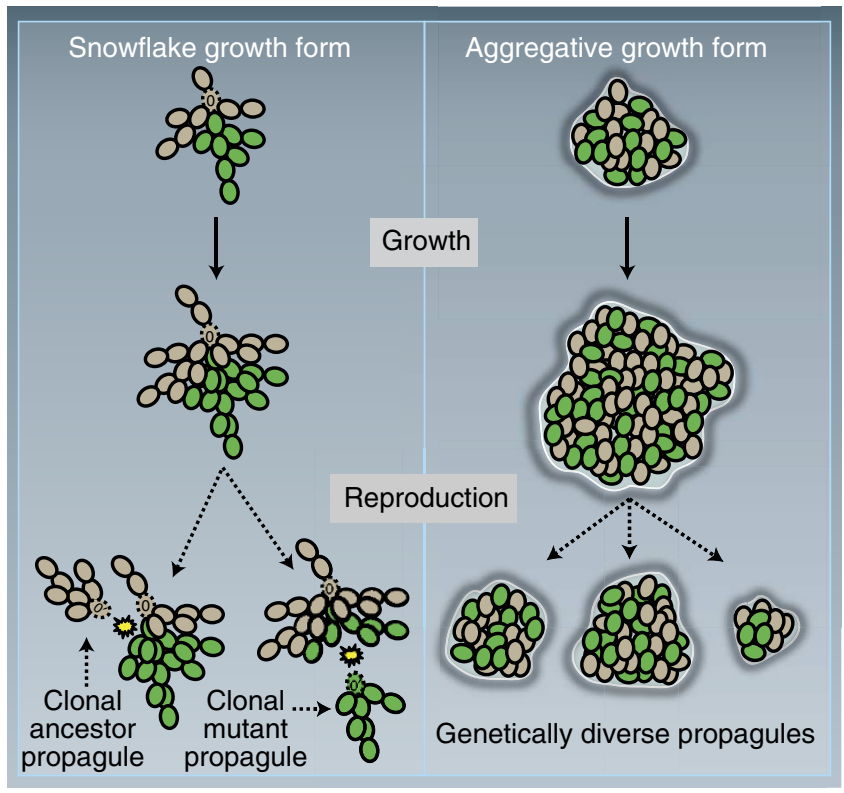

Figure 3 | Snowflake versus aggregate life cycles. Segregation of de novo mutations into mutant-only clusters is inevitable in snowflake yeast, but unlikely in aggregates. Shown are the chimeric clusters containing wild-type (tan) and mutant (green) lineages. Snowflake clusters reproduce by severing a single cell-cell connection (cells with a dashed outline marked $\mathrm{O}^{\prime}$ ), resulting in a unicellular genetic bottleneck. Only two types of propagule can be generated: those with an ancestral cell at the bottleneck (resulting in a purely ancestral propagule) or those with a mutant cell at the bottleneck (resulting in a purely mutant propagule). In contrast, the aggregative life cycle has no bottleneck. Instead, propagules are formed by randomly selecting cells from within the cluster.
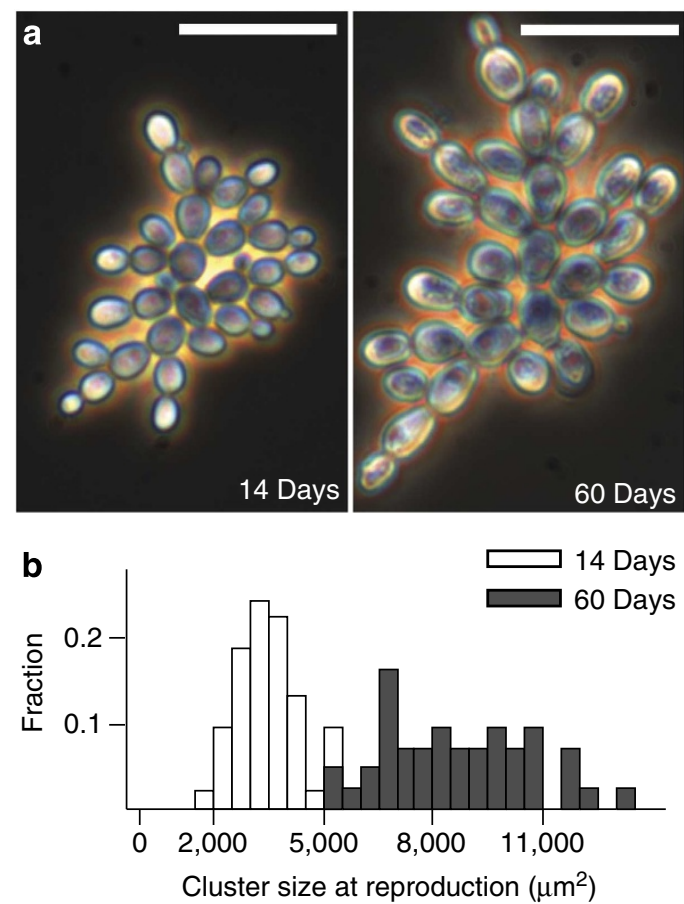

Figure 4 | Change in cell-level traits affects cluster-level traits. (a) By 60 days, cells have evolved to be 2.2-fold larger than an early snowflake yeast from the same population (14 days). This increases cluster size and settling rate. Both clusters are shown at the same magnification. Scale bars, $50 \mu \mathrm{m}$. (b) These large-celled yeast have evolved to produce propagules when 2.5 -fold larger than the 14-day strain, on average. The broad-sense heritability of this life history trait is 0.84 . phenotypic effects of all de novo mutations to be subject to selection.

Heritability of a key multicellular life history trait. We measured the heritability of a key multicellular life history trait in snowflake yeast, cluster size at reproduction, for strains isolated after either 14 or 60 transfers from the same population (shown in Fig. 4a). For each strain, we performed seven life history analyses, measuring the size of parent and offspring clusters at reproduction over $\sim 12 \mathrm{~h}$ of growth (Fig. $4 \mathrm{~b}$ ). The broad-sense heritability of this trait was 0.84 , which is extraordinarily high even by the standards of extant clonally reproducing multicellular organisms. For example, the $H^{2}$ of six life history traits of the colonial ascidian Botryllus schlosser range from 0.2 to 0.75 (ref. 56) and the average $H^{2}$ of five life history traits of Daphnia obtuse ranged from 0.35 to 0.47 , with a maximum of 0.83 (ref. 57). The snowflake developmental pattern creates clusters with high heritability by minimizing phenotypic variation among clusters of the same clone.

\section{Discussion}

Snowflake yeast evolve in as little as 7 days from a unicellular ancestor ${ }^{47}$. Snowflake yeast result from a single mutation (loss of the transcription factor ACE2, Fig. 1), which causes a simple change in the growth form of their unicellular ancestor: cells produced mitotically remain attached to their mother cells rather than separating. The snowflake developmental pattern (Fig. 2) creates clusters predisposed to multicellular adaptation. Clusters readily reproduce, creating offspring by fragmentation when tension among cells in the cluster exceeds the tensile strength of cellular adhesion ${ }^{5}$. Every time a new propagule separates from its parent cluster, it goes through a unicellular genetic bottleneck 
a
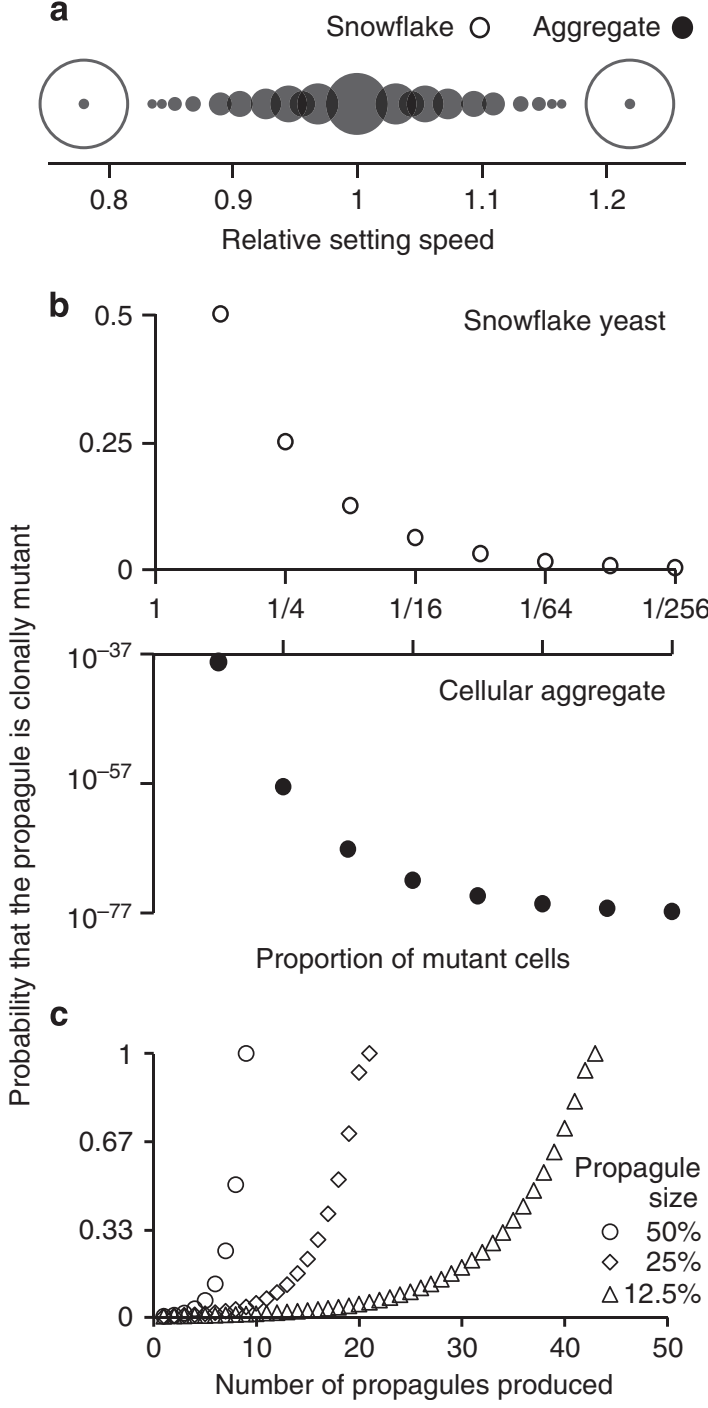

Figure 5 | The snowflake yeast body plan ensures genetic segregation. (a) We model the settling rate of propagules produced by 16-celled snowflake yeast clusters (open circles) or cellular aggregates (filled circles) that contain $50 \%$ small and large cells. Snowflake yeast produce offspring in which small- and large-cell alleles are completely segregated into different clusters (resulting in either slow or fast settling speed), while most aggregates contain a mixture of both cell types. (b) We model the probability that a propagule produced by a 256-cell cluster containing both wild-type cells and a mutant lineage will contain only mutant cells as a function of mutant frequency in the cluster. Snowflake yeast clusters have a far higher probability of producing mutant-only propagules than aggregate clusters. (c) Rare mutants in 256-cell snowflake yeast clusters initially have a low probability of forming their own propagules, but mutant-only propagules are eventually assured.

(Fig. 3). As a result, snowflake yeast clusters are typically uniclonal, nullifying the potential for within-group genetic conflict to erode multicellular complexity $1,16,20-26$. This does not require any anticheating adaptations (for examples, see refs $37,58)$, rather it is a geometric consequence of the snowflake yeast body plan. In addition to limiting genetic conflict, segregation of genetic variation among clusters also increases the multicellular evolvability by exposing the multicellular phenotype of novel mutations to selection (Fig. 4). Clusters of the same clone are physiologically similar (for example, similar cell size and shape, cell-cell adhesive strength, frequency of apoptosis, identical growth form and so on), minimizing within-clone variability and increasing the heritability of multicellular traits (Fig. 5). This means that cluster-level selection can cause rapid evolutionary change, and can act on mutations with relatively small multicellular-level phenotypic effects. These experiments demonstrate that a single genetic change not only can create a new level of biological organization, but can also facilitate the transition to higher-level individuality by potentiating higherlevel evolvability.

The evolution of multicellularity reveals how microevolutionary processes of selection and adaptation can cause macroevolutionary phenotypic changes. Newly evolved multicellular individuals are not yet optimized by prior selection; as a result, mutations having dramatic phenotypic effects are more likely to be beneficial than previously expected ${ }^{59,60}$. This observation helps to reconcile a long-standing debate in biology on the apparent incommensurability between macro- and microevolution and their relative importance in biological diversity ${ }^{61-63}$. Further, the specific mode of multicellularity evolved in our experiments reveals how novel, higher-level evolvability can readily arise through evolution. Snowflake multicellularity provides a key evolutionary benefit, unicellular genetic bottlenecks, which should be achievable by any organism in which clonal daughters attach just to their mothers. Once cluster growth meets volumetric constraints (which occur even with optimal packing of cells in a sphere ${ }^{64}$ ), further growth results in cell-cell scission, producing a propagule in which the oldest cell in the cluster is the genetic bottleneck. As a result, even simple clusters may be primed for higher-level adaptation. The exceptional evolvability of multicellular snowflake yeast arises as a consequence of the evolution of multicellularity, rather than selection for evolvability itself, sidestepping potential hurdles for its selection ${ }^{11,12}$.

\section{Methods}

RNA-seq. To prepare cells for RNA extraction, all yeast were inoculated 1:100 into $10 \mathrm{ml}$ Yeast Peptone Dextrose medium (YPD; per l: $20 \mathrm{~g}$ glucose, $20 \mathrm{~g}$ peptone, $10 \mathrm{~g}$ yeast extract), grown for $24 \mathrm{~h}$, then diluted 1:100 into fresh YPD and grown for $8 \mathrm{~h}$. Growth conditions were as described in ref. 5. Total RNA was extracted with the Qiagen RNeasy mini kit and then libraries were constructed using the Illumina TruSeq kit. RNA was quantified via fluorimetry using the RiboGreen assay, and RNA integrity was assessed using capillary electrophoresis on the Agilent BioAnalyzer 2100. Libraries were created, size selected and validated by staff at the University of Minnesota Genomics Center (UMGC). Using the Illumina HiSeq 2000, 10 million 50 bp PE reads were collected per sample. Reads were mapped to the Y55 genome (Sanger) to identify single-nucleotide polymorphisms in coding regions, and the $\mathrm{S} 288 \mathrm{C}$ genome for expression analysis (mapping done in CLC Genomics Workbench v. 6.1). Gene expression was measured as reads mapped per kilobase of exon per million reads mapped (RPKM) ${ }^{65}$. RKPM values were then $\log _{2}$ transformed for graphical display in R. Gene expression was considered significantly different between unicellular and 7-day multicellular strains when overall false discovery rate-corrected $P$ values $<0.05$ (ref. 66). Genes with very low expression levels $(\mathrm{RPKM}<1)$ were excluded. Gene ontology enrichment analysis of differentially expressed genes was conducted using GeneCodis3 (refs 67,68).

Sequencing ACE2. Genomic DNA was extracted using the Zymo YeaStar kit from one snowflake yeast isolate derived from each of the 10 replicate populations described in Ratcliff et al. ${ }^{5}$ ACE2 (414 bp upstream and $433 \mathrm{bp}$ downstream of the CDS, primers available on request) was PCR amplified and Sanger sequenced at the UMGC.

Transformation. To test the hypothesis that the loss of ACE2 was sufficient to generate the snowflake phenotype, we knocked out ACE2 in our unicellular ancestor (strain Y55) through replacement of the ACE2 ORF with NATMX4 using the LiAc/SS-DNA/PEG method of transformation ${ }^{69}$. ace2::NATMX4/ ace 2::NATMX4 homozygotes were generated by the autodiploidization of single spores dissected from tetrads formed by heterozygous transformants; tetrads were dissected onto YPD and then ace2 $\triangle N A T M X 4 / a c e 2 \triangle N A T M X 4$ diploids were selected by replica plating to YPD with $100 \mathrm{mgl}^{-1}$ nourseothricin (obtained from Werner Bioagents, Jena, Germany). The genotype was confirmed by PCR, as well as ensuring that all four spores of the selected isolate were nourseothricin resistant. To confirm that the loss of $A C E 2$ functionality is responsible for the snowflake yeast growth form in our experimentally evolved yeast, we functionally 
complemented an isolate obtained after 7 days of selection (population 1) with the ancestral ACE2 allele. To accomplish this, we first inserted a KANMX4 cassette downstream of the Y55 ACE2 $3^{\prime}$ UTR (position 404446 on chromosome 7). We then replaced a single copy of the 7-day snowflake yeast with this ACE2-KANMX fusion via the LiAc/SS-DNA/PEG method of transformation, plating cells on YPD with $200 \mathrm{mg}$ G418/L.

Testing the model of the snowflake yeast body plan. To assess our model's description of snowflake yeast growth form, we compared the predicted number of cells of each distance from the basal cell $(0,1,2,3 \ldots x)$ with experimentally obtained counts. To generate this prediction, we calculated the number of complete doublings from the basal cell, $d$, as $\log _{2}$ (cell number), rounded down. We also calculated $s$, the fraction of cells within the cluster that reproduced from the last complete doubling, as described above. Inputting values of $d$ and $s$ into our model, we generated the expected number of cells at each distance $x$ from the basal cell. We then compared the actual number of cells at each distance from the basal cell, determined empirically (for example, Fig. 2c), with the number predicted by our model.

We tested predictions of our model against empirically obtained branching pattern data from 7-10 clusters of five strains of snowflake yeast, isolated after either $7,14,28,65$ or 227 days of experimental evolution. On days 28 and 65 , the intensity of settling selection was increased to continue selecting for larger cluster size (see ref. 47) for a complete description of the experiment). Clusters were grown from single cells that were obtained via enzymatic digestion with lyticase and $\beta$-glucuronidase as described in ref. 5. Clusters were grown in YPD medium, at $30^{\circ} \mathrm{C}$ with 250 r.p.m. shaking, and then imaged once after $6 \mathrm{~h}$ of growth. All microscopy was performed on an Olympus IX70 inverted microscope with a Scion CFW-1310C camera. We empirically determined each cell's distance from the basal (seed) cell. See the inset of Fig. $2 c$ for an example of a cluster with this distance annotated.

Calculating broad-sense heritability of cluster size at reproduction. For each strain, we performed seven life history analyses, measuring the size of parent and offspring clusters at reproduction over $\sim 12 \mathrm{~h}$ of growth. Following the procedure of van Kleunen et al. ${ }^{70}, H^{2}$ was calculated using REML analysis of variance to parse the variance components (VC) of genotype and replicate LH analysis effects: $H^{2}=V C_{\text {genotype }} /\left(V C_{\text {genotype }}+V C_{\mathrm{LH}}\right.$ analysis replicate $\left.+V C_{\text {residual }}\right)$, with $\mathrm{LH}$ analysis replicate (a random effect) nested in strain (a random effect).

Quantifying cluster size at reproduction. Time-lapse movies were conducted following the protocol described in ref. 5. In brief, individual clusters from a singlestrain isolate (put through three rounds of single-colony isolation) were inoculated into 0.5 - $\mu$ l droplets in Nunc Lab-Tek II chambered cover glass slides. To limit evaporation, $10 \mu \mathrm{l}$ of water was placed on the walls of the chamber and the chamber sealed with clear tape. Illumination was kept to a minimum to avoid heating the sample. Images were acquired every minute for $16 \mathrm{~h}$. We determined the cluster size at reproduction by measuring the two-dimensional (top-down) footprint of the cluster one frame before the propagule separated in ImageJ.

Statistical analysis. All statistical tests were conducted in JMP 9.0. Assumptions of parametric tests were checked before use.

\section{References}

1. Maynard Smith, J. \& Szathmáry, E. The Major Transitions in Evolution (Oxford Univ. Press, 1995)

2. Bonner, J. T. The origins of multicellularity. Integr. Biol. 1, 27-36 (1998).

3. Okasha, S. Multilevel selection and the major transitions in evolution. Philos. Sci. 72, 1013-1025 (2005).

4. Michod, R. On the transfer of fitness from the cell to the multicellular organism. Biol. Philos. 20, 967-987 (2005).

5. Ratcliff, W. C., Denison, R. F., Borrello, M. \& Travisano, M. Experimental evolution of multicellularity. Proc. Natl Acad. Sci. USA 109, 1595-1600 (2012).

6. Libby, E. \& Rainey, P. B. A conceptual framework for the evolutionary origins of multicellularity. Phys. Biol. 10, 035001 (2013).

7. Rainey, P. B. \& Kerr, B. Cheats as first propagules: a new hypothesis for the evolution of individuality during the transition from single cells to multicellularity. Bioessays 32, 872-880 (2010).

8. Blackstone, N. W. Why did eukaryotes evolve only once? Genetic and energetic aspects of conflict and conflict mediation. Philos. Trans. R Soc. B: Biol. Sci. 368, 20120266 (2013).

9. Okasha, S. Evolution and the Levels of Selection (ed. ebrary, I.) (Oxford Univ. Press, 2006).

10. Hamilton, A. \& Fewell, J. in Groups, Indviduals, and the Emergence of Sociality: The Case for Division of Labor (eds Bouchard, F. \& Huneman, P.) 175-194 (The MIT Press, 2013).

11. Pigliucci, M. Is evolvability evolvable? Nat. Rev. Genet. 9, 75-82 (2008).

12. Godfrey-Smith, P. Darwinian Populations and Natural Selection (Oxford Univ. Press, 2009).
13. Lewontin, R. C. The units of selection. Annu. Rev. Ecol. Syst. 1, 1-18 (1970). 14. Griesemer, J. The units of evolutionary transition. Selection 1, 67-80 (2001).

15. Michod, R. E. \& Herron, M. D. Cooperation and conflict during evolutionary transitions in individuality. J. Evol. Biol. 19, 1406-1409 (2006).

16. Grosberg, R. K. \& Strathmann, R. R. One cell, two cell, red cell, blue cell: the persistence of a unicellular stage in multicellular life histories. Trends Ecol. Evol. 13, 112-116 (1998)

17. Diggle, S. P., Griffin, A. S., Campbell, G. S. \& West, S. A. Cooperation and conflict in quorum-sensing bacterial populations. Nature 450, 411-414 (2007)

18. Velicer, G. J., Kroos, L. \& Lenski, R. E. Developmental cheating in the social bacterium Myxococcus xanthus. Nature 404, 598-600 (2000).

19. Gilbert, O., Foster, K., Mehdiabadi, N., Strassmann, J. \& Queller, D. High relatedness maintains multicellular cooperation in a social amoeba by controlling cheater mutants. Proc. Natl Acad. Sci. USA 104, 8913-8917 (2007).

20. Ostrowski, E. A. \& Shaulsky, G. Learning to get along despite struggling to get by. Genome Biol. 10, 218 (2009).

21. Buss, L. W. The Evolution of Individuality (Princeton Univ. Press, 1987).

22. Roze, D. \& Michod, R. E. Mutation, multilevel selection, and the evolution of propagule size during the origin of multicellularity. Am. Nat. 158, 638-654 (2001).

23. Folse, H. J. Evolution and Individuality; Beyond the Genetically Homogeneous Organism (Stanford University, 2011)

24. Folse, H. J. \& Roughgarden, J. What is an individual organism? A multilevel selection perspective. Q Rev. Biol. 85, 447-472 (2010).

25. Dawkins, R. The Selfish Gene (Oxford Univ. Press, 1976).

26. Strathmann, R. R. Functional Design in the Evolution of Embryos and Larvae (Seminars in Cell and Developmental Biology Ser. 11, Academic Press, c1996-, 2000).

27. Queller, D. C. Cooperators since life began. Q Rev. Biol. 72, 184-188 (1997).

28. Williams, G. W. Natural Selection: Domains, Levels, and Challenges (Oxford Univ. Press, 1992).

29. Damore, J. A. \& Gore, J. Understanding microbial cooperation. J. Theor. Biol. 299, 31-41 (2012).

30. Dunn, C. W. \& Wagner, G. P. The evolution of colony-level development in the Siphonophora (Cnidaria: Hydrozoa). Dev. Genes Evol. 216, 743-754 (2006).

31. Ratcliff, W. C. et al. Experimental evolution of an alternating uni-and multicellular life cycle in Chlamydomonas reinhardtii. Nat. Commun. 4, 2742 (2013).

32. Sucgang, R. et al. Comparative genomics of the social amoebae Dictyostelium discoideum and Dictyostelium purpureum. Genome Biol. 12, R20 (2011).

33. Shimkets, L. J. in Myxobacteria II (eds Dworkin, M. \& Kaiser, D.) 85-107 (American Society for Microbiology, 1993).

34. Fiegna, F., Yu, Y. T., Kadam, S. V. \& Velicer, G. J. Evolution of an obligate social cheater to a superior cooperator. Nature 441, 310-314 (2006).

35. Kuzdzal-Fick, J. J., Fox, S. A., Strassmann, J. E. \& Queller, D. C. High relatedness is necessary and sufficient to maintain multicellularity in Dictyostelium. Science 334, 1548-1551 (2011).

36. Khare, A. et al. Cheater-resistance is not futile. Nature 461, 980-982 (2009).

37. Manhes, P. \& Velicer, G. J. Experimental evolution of selfish policing in social bacteria. Proc. Natl Acad. Sci. USA 108, 8357-8362 (2011).

38. Queller, D. C., Ponte, E., Bozzaro, S. \& Strassman, J. E. Single-gene greenbeard effects in the social amoeba Dictyostelium discoideum. Science 299, 105-106 (2003).

39. D'Souza, G. et al. Less is more: selective advantages can explain the prevalent loss of biosynthetic genes in bacteria. Evolution 68, 2559-2570 (2014).

40. Harcombe, W. Novel cooperation experimentally evolved between species. Evolution 64, 2166-2172 (2010).

41. Hillesland, K. L. \& Stahl, D. A. Rapid evolution of stability and productivity at the origin of a microbial mutualism. Proc. Natl Acad. Sci. USA 107, 2124-2129 (2010).

42. Overmann, J. in Recent Advances in Phototrophic Prokaryotes (ed. Hallenbeck, P. C.) 15-29 (Springer, 2010).

43. Werth, S. \& Scheidegger, C. Congruent genetic structure in the lichen-forming fungus Lobaria pulmonaria and its green-algal photobiont. Mol. Plant Microbe Interact. 25, 220-230 (2012).

44. Herre, E. A., Knowlton, N., Mueller, U. G. \& Rehner, S. A. The evolution of mutualisms: exploring the paths between conflict and cooperation. Trends Ecol. Evol. 14, 49-53 (1999).

45. Eshel, I. \& Cavalli-Sforza, L. L. Assortment of encounters and evolution of cooperativeness. Proc. Natl Acad. Sci. USA 79, 1331 (1982).

46. McFadden, G. I. Primary and secondary endosymbiosis and the origin of plastids. J. Phycol. 37, 951-959 (2001).

47. Ratcliff, W. C., Pentz, J. T. \& Travisano, M. Tempo and mode of multicellular adaptation in experimentally-evolved Saccharomyces cerevisiae. Evolution 67, 1573-1581 (2013).

48. King, L. \& Butler, G. Ace2p, a regulator of CTS1 (chitinase) expression, affects pseudohyphal production in Saccharomyces cerevisiae. Curr. Genet. 34, 183-191 (1998). 
49. O’Conalláin, C., Doolin, M., Taggart, C., Thornton, F. \& Butler, G. Regulated nuclear localisation of the yeast transcription factor Ace2p controls expression of chitinase (CTS1) in Saccharomyces cerevisiae. Mol. Gen. Genet. 262, 275-282 (1999).

50. McBride, H. J., Yu, Y. \& Stillman, D. J. Distinct regions of the Swi5 and Ace2 transcription factors are required for specific gene activation. J. Biol. Chem. 274, 21029-21036 (1999).

51. Voth, W. P., Olsen, A. E., Sbia, M., Freedman, K. H. \& Stillman, D. J. ACE2, CBK1, and BUD4 in budding and cell separation. Eukaryot. Cell 4, 1018-1028 (2005).

52. Oud, B. et al. Genome duplication and mutations in ACE2 cause multicellular, fast-sedimenting phenotypes in evolved Saccharomyces cerevisiae. Proc. Natl Acad. Sci. 110, E4223-E4231 (2013).

53. Klein, H. L. \& Petes, T. D. Intrachromosomal gene conversion in yeast. Nature 289, 144-148 (1981).

54. Maynard Smith, J. Evolutionary progress and levels of selection. Evol. Prog. 219, 230 (1988).

55. Barton, N. \& Partridge, L. Limits to natural selection. Bioessays 22, 1075-1084 (2000).

56. Yund, P. O., Marcum, Y. \& Stewart-Savage, J. Life-history variation in a colonial ascidian: broad-sence heritabilities and tradeoffs in allocation to asexual growth and male and female reproduction. Biol. Bull. 192, 290-299 (1997).

57. Spitze, K. Population structure in Daphnia obtusa: quantitative genetic and allozymic variation. Genetics 135, 367-374 (1993).

58. Travisano, M. \& Velicer, G. J. Strategies of microbial cheater control. Trends Microbiol. 12, 72-78 (2004).

59. Fisher, R. A. in The Genetical Theory of Natural Selection (ed. Bennett, H.) (Oxford Univ. Press, 1930).

60. Goldschmidt, R. B. Theoretical Genetics (Univ. California Press, 1958).

61. Lenski, R. E. \& Travisano, M. Dynamics of adaptation and diversification: a 10,000-generation experiment with bacterial populations. Proc. Natl Acad. Sci. USA 91, 6808-6814 (1994).

62. Simpson, G. G. in Tempo and Mode in Evolution (eds Fitch, W. M. \& Ayala, F. J.) (Columbia Univ. Press, 1944).

63. Gould, S. J. \& Eldredge, N. Punctuated equilibria: the tempo and mode of evolution reconsidered. Paleobiology 3, 115-151 (1977).

64. Libby, E., Ratcliff, W. C., Travisano, M. \& Kerr, B. Geometry shapes evolution of early multicellularity. PLoS Comput. Biol. 10, e1003803 (2014).

65. Carver, T. et al. BamView: visualizing and interpretation of next-generation sequencing read alignments. Brief. Bioinform. 14, 203-212 (2013).

66. Baggerly, K. A., Deng, L., Morris, J. S. \& Aldaz, C. M. Differential expression in SAGE: accounting for normal between-library variation. Bioinformatics 19, 1477-1483 (2003).

67. Carmona-Saez, P., Chagoyen, M., Tirado, F., Carazo, J. M. \& Pascual-Montano, A. GENECODIS: a web-based tool for finding significant concurrent annotations in gene lists. Genome Biol. 8, R3 (2007).
68. Nogales-Cadenas, R. et al. GeneCodis: interpreting gene lists through enrichment analysis and integration of diverse biological information. Nucleic Acids Res. 37, W317-W322 (2009).

69. Gietz, R. D., Schiestl, R. H., Willems, A. R. \& Woods, R. A. Studies on the transformation of intact yeast cells by the LiAc/SS-DNA/PEG procedure. Yeast 11, 355-360 (1995).

70. Kleunen, M. v., Fischer, M. \& Schmid, B. Experimental life-history evolution: selection on the allocation to sexual reproduction and its plasticity in a clonal plant. Evolution 56, 2168-2177 (2002).

\section{Acknowledgements}

This paper is dedicated to the memory of Johnathon Fankhauser. JF (as he was called in the lab) was a tremendously talented student and colleague who flung himself whole heartedly into every endeavour. We miss him greatly. We thank Jessica Nguyen, Chelsea Du Fresne and Kristin Jacobsen for lab assistance; Jennifer Pentz, Ruth Shaw and Georgiana May for feedback on the manuscript; and Ben Kerr and Amanda Muehlbauer for mathematical advice. This work was supported by NSF DEB-1051115 (W.C.R., M.T., J.D.F.), NSF GRFP no. 00006595 (J.D.F.) and the Max Planck Institute for Evolutionary Biology (W.C.R., D.W.R., D.G.).

\section{Author contributions}

W.C.R. and M.T. conceived of the project. J.D.F. performed and analysed the RNA/DNA sequencing. D.W.R. and D.G. helped W.C.R. plan and execute the genetic transformations. W.C.R. conducted the remaining experiments, analysed their data, performed the modelling and wrote the first draft of the paper (all authors, especially M.T., provided constructive feedback)

\section{Additional information}

Supplementary Information accompanies this paper at http://www.nature.com/ naturecommunications

Competing financial interests: The authors declare no competing financial interests.

Reprints and permission information is available online at http://npg.nature.com/ reprintsandpermissions/

How to cite this article: Ratcliff, W. C. et al. Origins of multicellular evolvability in snowflake yeast. Nat. Commun. 6:6102 doi: 10.1038/ncomms7102 (2015).

(c) (i) This work is licensed under a Creative Commons Attribution 4.0 International License. The images or other third party material in this article are included in the article's Creative Commons license, unless indicated otherwise in the credit line; if the material is not included under the Creative Commons license, users will need to obtain permission from the license holder to reproduce the material To view a copy of this license, visit http://creativecommons.org/licenses/by/4.0/ 